\title{
Susceptor Coupling for the Uniformity and Dopant Activation Efficiency in Implanted Si Under Fixed-Frequency Microwave Anneal
}

\author{
Yao-Jen Lee, Fu-Kuo Hsueh, Michael I. Current, Ching-Yi Wu, and Tien-Sheng Chao
}

\begin{abstract}
Microwave annealing of dopants in Si has been reported to produce highly activated junctions at temperatures far below those needed for comparable results using conventional thermal processes. However, during conventional fixed-frequency microwave heating, standing wave patterns can be established in the microwave processing chamber, resulting in nodes and antinodes over the processing area, resulting in thermal variations over the process wafer. In this letter, the effects of $\mathrm{Si}$ or quartz susceptor wafers on dopant activation and sheet resistance uniformity during fixed-frequency microwave anneal are studied. The composition, number, and spacing of susceptor wafers were varied in a systematic fashion in these experiments.
\end{abstract}

Index Terms-Coupling effect, low temperature, microwave anneal, phosphorus, quartz.

\section{INTRODUCTION}

$\mathbf{I}$ T IS expected that metal-oxide-semiconductor field-effect transistors will scale to under $17 \mathrm{~nm}$ by 2015 to meet the International Technology Roadmap for Semiconductors 2009 [1]. A key technical challenge is to form highly activated doped junctions with depths of less than $10 \mathrm{~nm}$. In order to avoid dopant diffusion effects, which are dominant at high anneal temperatures, low-temperature annealing with microwave excitation appears to offer promise. Previous studies [2]-[5] of the microwave annealing with temperatures around $400{ }^{\circ} \mathrm{C}-480{ }^{\circ} \mathrm{C}$ have shown good effectiveness for suppression of dopant diffusion with high dopant activation efficiency.

In this letter, the coupling effects of $\mathrm{Si}$ and quartz susceptor wafers to improve the uniformity and dopant activation effi-

Manuscript received October 20, 2011; revised October 30, 2011; accepted November 8, 2011. Date of publication December 15, 2011; date of current version January 27, 2012. This work was supported in part by the National Science Council, Taiwan, under Contracts NSC-99-2221-E-492-031-MY2 and NSC-98-2221-E-492-021-MY3. The review of this letter was arranged by Editor C.-P. Chang.

Y.-J. Lee is with the National Nano Device Laboratories, Hsinchu 30010, Taiwan, and also with the Department of Physics, National Chung Hsing University, Taichung 402, Taiwan (e-mail: yjlee@ndl.narl.org.tw).

F.-K. Hsueh is with the National Nano Device Laboratories, Hsinchu 30010, Taiwan, and also with the Department of Electrophysics, National Chiao Tung University, Hsinchu 30050, Taiwan.

M. I. Current is with Current Scientific, San Jose, CA 95124 USA.

C.-Y. Wu is with Bayzu Precision Company, Tainan 74144, Taiwan.

T.-S. Chao is with the Department of Electrophysics, National Chiao Tung University, Hsinchu 30050, Taiwan.

Color versions of one or more of the figures in this letter are available online at http://ieeexplore.ieee.org.

Digital Object Identifier 10.1109/LED.2011.2176100 ciency for fixed-frequency microwave annealing process are investigated. In other studies [6], the more complex variablefrequency microwave methods to improve thermal uniformity have been proposed and studied. In this fixed-frequency study, the composition, number, and spacing of susceptor wafers placed around a single Si process wafer are investigated.

\section{EXPERIMENTS}

One-hundred-fifty-millimeter p-type Si (100) substrates with a resistivity of $10-30 \Omega \cdot \mathrm{cm}$ were used. The samples were implanted by phosphorus $\left({ }^{31} \mathrm{P}\right)$ at $15 \mathrm{keV}$ with a dose of $1 \times 10^{15} \mathrm{ions} / \mathrm{cm}^{2}$ at room temperature. To minimize ion channeling effects, all wafers were oriented during implantation with their surface normal at $7^{\circ}$ from the incident beam and with a $22^{\circ}$ in-plane twist. All splits were annealed by microwave for $100 \mathrm{~s}$ with the same power, where the microwave frequency was fixed at $5.8 \mathrm{GHz}$. Wafer temperatures during the microwave anneal were monitored by a pyrometer with a direct line of sight to the lower surface of the wafer in the chamber. Before the microwave annealing process, a 30-min nitrogen purge was performed and maintained throughout the anneal cycle. Microwave power generated by magnetrons was around $2100 \mathrm{~W}$. In addition, 150-mm p-type $\mathrm{Si}$ (100) substrates with a resistivity of $10-30 \Omega \cdot \mathrm{cm}$ and $150-\mathrm{mm}$ quartz substrates were used as susceptors to improve the $R s$ uniformity and dopant activation efficiency. Different chamber configurations were summarized in Table I. The distance between adjacent slots was $1 \mathrm{~cm}$. In addition, the split for $1000{ }^{\circ} \mathrm{C}$ spike RTA has also been included. Sheet resistance $(R s)$ was measured by a contact four-point probe (4PP) and noncontact carrier spreading analysis using junction photovoltage (JPV) measurements for sheet resistance and junction leakage (RsL) [7]. SIMS dopant profiles and crosssectional TEM images were also studied.

\section{RESUlts And Discussions}

Fig. 1 shows the SIMS profiles of the ${ }^{31} \mathrm{P}$ concentrations. The dopant profile after the low-temperature microwave anneals was indistinguishable from the as-implanted profiles, indicating no dopant diffusion motion for these low-temperature anneals. In addition, the insert TEM pictures depict the as-implanted and postannealed samples. The amorphous region can be distinguished as the lightly shaded area. The cross-sectional TEM image of the implanted sample after microwave annealing for $100 \mathrm{~s}$ shows the excellent crystallinity of the regrown Si layer. 
TABLE I

Different Chamber Configurations For THE COMPARISONS of Coupling Effects. The Distance Between Adjacent SLOTS WAS $1 \mathrm{~cm}$ ONLY. P.W. IS PROCESS WAFER, S.W. IS Si SUSCEPTOR, AND Q.W. IS QUARTZ SUSCEPTOR

\begin{tabular}{|c|l|l|l|l|l|l|l|}
\hline configuration & Split 1 & Split 2 & Split 3 & Split 4 & Split 5 & Split 6 & Split 7 \\
\hline Slot 1 & & & & & S.W. & & \\
\hline Slot 2 & & & & & & & \\
\hline Slot 3 & & & S.W. & S.W. & & & Q.W. \\
\hline Slot 4 & & S.W. & S.W. & & & Q.W. & Q.W. \\
\hline Slot 5 & P.W. & P.W. & P.W. & P.W. & P.W. & P.W. & P.W. \\
\hline Slot 6 & & S.W. & S.W. & & & Q.W. & Q.W. \\
\hline Slot 7 & & & S.W. & S.W. & & & Q.W. \\
\hline Slot 8 & & & & & & & \\
\hline Slot 9 & & & & & S.W. & & \\
\hline
\end{tabular}

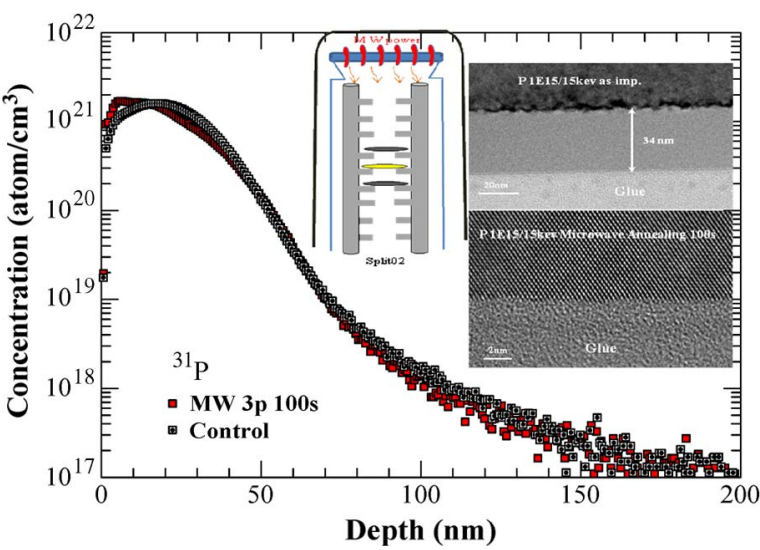

Fig. 1. SIMS profiles of $\mathrm{P}$ concentration at a dose of $1 \times 10^{15}$ ions $/ \mathrm{cm}^{2}$. The microwave anneal process resulted in no significantly dopant diffusion. The inserts were the cross-sectional TEM pictures of as-implanted and after 100-s microwave annealing and the schematic describing the microwave annealing setup.

The kinetics and mechanisms of the crystallinity regrowth during microwave annealing were solid-phase epitaxy, nucleating at the $\mathrm{a} / \mathrm{c}$ interface, and proceeding toward the sample surface.

The sheet resistances of all splits with in-wafer uniformities (error bars) are summarized in Fig. 2. There are 49 points measured for each split by 4PP and 973 points per RsL map. The average $R s$ of split 1 , with a single $\mathrm{Si}$ process wafer, was close to $580 \Omega /$ sq. The $R s$ variations of split 1 were very large, and standard deviation was up to $135.8 \Omega /$ sq. However, if one added two Si susceptors inside the chamber and above and below the process wafer, the average $R s$ of split 2 was close to $240 \Omega$ /sq with standard deviation improving to $14.7 \Omega /$ sq. This indicates that optimized coupling effects improve the uniformity and increase the dopant activation efficiency simultaneously. From the results of splits 1 and 2, although $\mathrm{Si}$ susceptor wafers could soak up the microwave power inside the chamber, the microwave coupling effects between $\mathrm{Si}$ susceptors and process wafers would be an important factor to increase the dopant activation efficiency.

As additional $\mathrm{Si}$ susceptors are added or the spacing increased (splits 3-5), Rs with four $\mathrm{Si}$ susceptors increased to

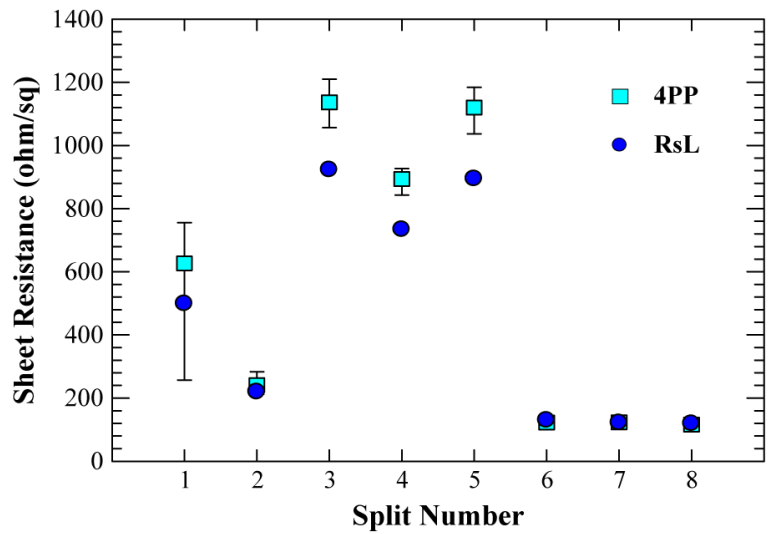

Fig. 2. Sheet resistance values, measured by 4PP and a JPV carrier spreading technique, RsL, for the wafer splits in this study. The position and composition of the susceptors around the Si test wafer (shown as a filled rectangle) are shown above the $R s$ values. In addition, split 8 is for $1000{ }^{\circ} \mathrm{C}$ spike RTA.

$\approx 1120 \Omega /$ sq with a standard deviation of up to $44.5 \Omega / \mathrm{sq}$, which indicates that the sharing of microwave power by four Si susceptors dominates the dopant activation efficiency.

On the other hand, the distance between process wafer and Si susceptor wafers is also an important factor of the coupling effects. As the distance between a pair of $\mathrm{Si}$ susceptors and process wafer is increased from one to two units, split 4 , and to three units, split 5 , the $R s$ remains high, near $1000 \Omega$ /sq with a standard deviation of $\approx 30 \Omega /$ sq. The temperature profiles versus annealing time by microwave are shown in Fig. 3. For split 1 , with only one process wafer inside the chamber, the temperature ramping rate is the fastest as compared with those of other splits. However, as the temperature saturated, the temperature profile was unstable, which indicates that the standing wave patterns were established in the microwave processing chamber, resulting in nodes and antinodes over the process wafer, causing an unstable temperature profile. As one adds two $\mathrm{Si}$ wafers as the susceptors, split 2 , the temperature rampingup slope decreases as compared with that of split 1 because the $\mathrm{Si}$ susceptors would soak up the microwave power inside the chamber. In addition, as one increases the distance between Si susceptors and process wafer from one to two units, split 4, the temperature ramping-up rate was the same as that of split 2 but with lower maximum temperature, by $20^{\circ} \mathrm{C}$, for split 4 , as compared with split 2.

Microwave power absorption of solid materials is due to their magnetic, dielectric, and conductivity properties. The loss factor $\varepsilon^{\prime \prime}$ determines the absorption of the propagating electric field. In addition, $\varepsilon^{\prime \prime}$ is the material parameter that conveys the effects of conduction and polarization loss mechanisms during microwave heating of doped silicon. The power absorbed during microwave annealing is described by

$$
P_{\mathrm{abs}}=\omega \varepsilon_{0} \varepsilon^{\prime \prime}|E|^{2}
$$

where $P_{\text {abs }}$ is the microwave power absorbed per unit volume, $\varepsilon^{\prime \prime}$ is the loss factor, $\omega$ represents the angular frequency of the microwaves, and $E$ is the root-mean-square internal electric field. The majority of $P_{\text {abs }}$ was transformed into heat inside the material and results in a temperature rise [8]. The microwave power absorbed by quartz susceptors was very small as 


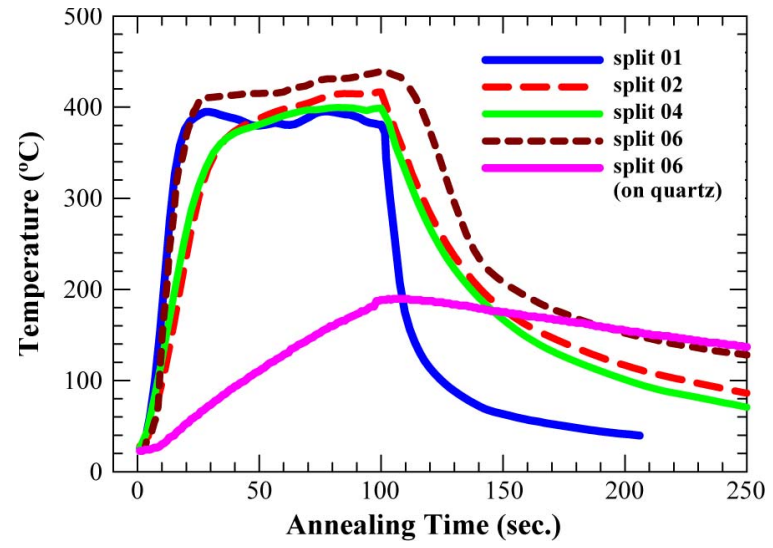

Fig. 3. Comparison of temperature profiles versus annealing time. The microwave annealing time was defined as the period when the microwave power was turned on.

compared with that by $\mathrm{Si}$ wafers. Therefore, as one adds susceptors inside the microwave chamber, $\mathrm{Si}$ susceptors also soak up the microwave power inside the chamber [8], but quartz susceptors absorb far less.

In Fig. 3, when quartz susceptors are used, in splits 6, the peak process $\mathrm{Si}$ wafer temperature was $445{ }^{\circ} \mathrm{C}$, yet low $R s$ values are measured with both the contact $4 \mathrm{PP}$ and the noncontact RsL method. Carrier recombination and trap-assisted tunneling leakage currents measured with the RsL tool [7] were quite modest, in the range of $1-10 \mu \mathrm{A} / \mathrm{cm}^{2}$, for all wafer splits in this study.

On the other hand, the maximum temperature of quartz wafers of split 6 was $180{ }^{\circ} \mathrm{C}$ only with low ramping-up and ramping-down rates, which was very low as compared with that of $\mathrm{Si}$ susceptors due to the low loss factor. Therefore, quartz wafers could prevent power of process $\mathrm{Si}$ wafer from dissipation during microwave annealing process with the highest peak temperature among all splits.

After microwave power was turned off, the ramping-down rates also depended on the number of $\mathrm{Si}$ and quartz susceptors. More susceptors slow down the ramping-down rate of temperature. Therefore, susceptors could stabilize the process wafer temperature by slowing the temperature ramping-down rate after microwave power turnoff.

\section{CONCLUSION}

In this letter, the effects of susceptor coupling on the dopant activation levels and uniformity of implant-doped Si for fixedfrequency microwave annealing have been investigated. The number and placement of various combinations of $\mathrm{Si}$ and quartz susceptor wafers changed the temperature profiles for 100-s microwave heating cycles as well as the sheet resistance level and uniformity. The addition of susceptor wafers in the near vicinity of the process wafer improved the sheet resistance uniformity and caused higher dopant activation level. The use of low-susceptibility quartz wafers resulted in remarkably low sheet resistance and low leakage current values.

\section{ACKNOWLEDGMENT}

The authors would like to thank the DSG Technologies for the useful suggestions and discussions.

\section{REFERENCES}

[1] [Online]. Available: www.itrs.net

[2] Y.-J. Lee, F.-K. Hsueh, S.-C. Huang, J. M. Kowalski, J. E. Kowalski, A. T. Y. Cheng, A. Koo, G.-L. Luo, and C.-Y. Wu, "A low-temperature microwave annealing process for boron-doped ultrathin Ge epilayer on Si substrate," IEEE Electron Device Lett., vol. 30, no. 2, pp. 123-125, Feb. 2009.

[3] Y.-J. Lee, S.-S. Chuang, F.-K. Hsueh, H.-M. Lin, S.-C. Wu, C.-Y. Wu, and T.-Y. Tseng, "Dopant activation in single crystalline germanium by low temperature microwave annealing," IEEE Electron Device Lett., vol. 32, no. 2, pp. 194-196, Feb. 2011.

[4] Y. J. Lee, Y. L. Lu, F. K. Hsueh, K. C. Huang, C. C. Wan, T. Y. Cheng, M. H. Han, J. M. Kowalski, J. E. Kowalski, D. Heh, H. T. Chuang, Y. Li, T. S. Chao, C. Y. Wu, and F. L. Yang, "3D $65 \mathrm{~nm}$ CMOS with $320{ }^{\circ} \mathrm{C}$ microwave dopant activation," in IEDM Tech. Dig., 2009, pp. 31-34.

[5] F.-K. Hsueh, Y.-J. Lee, K.-L. Lin, M. I. Current, C.-Y. Wu, and T.-S. Chao, "Amorphous layer regrowth and activation of $\mathrm{P}$ and As implanted Si by low-temperature microwave annealing," IEEE Trans. Electron Devices, vol. 58, no. 7, pp. 2088-2093, Jul. 2011.

[6] T. L. Alford, I. Ahmad, and R. Hubbard, "Variable frequency microwave induced low temperature dopant activation in ion implanted silicon," in Proc. 17th Int. Conf. Adv. Therm. Process. Semicond., RTP, 2009, pp. 1-5.

[7] V. N. Faifer, M. I. Current, T. M. H. Wong, and V. V. Souchkov, "Noncontact sheet resistance and leakage current mapping for ultra-shallow junctions," J. Vac. Sci. Technol. B, Microelectron. Nanometer Struct., vol. 24, no. 1, pp. 414-420, Jan./Feb. 2006.

[8] T. L. Alford, D. C. Thompson, J. W. Mayer, and N. D. Theodore, "Dopant activation in ion implanted silicon by microwave annealing," J. Appl. Phys., vol. 106, no. 11, p. 114902 , Dec. 2009. 